УДК 531.01

\title{
НОВЫЕ СЛУЧАИ ИНТЕГРИРУЕМЫХ СИСТЕМ ДЕВЯТОГО ПОРЯДКА С ДИССИПАЦИЕЙ
}

\author{
М. В. Шамолин \\ Представлено академиком РАН В.В. Козловым 03.10.2019 г.
}

Поступило 07.10.2019 г.

\begin{abstract}
В работе показана интегрируемость некоторых классов однородных по части переменных динамических систем девятого порядка, в которых выделяется система на касательном расслоении к четырёхмерным многообразиям. При этом силовые поля обладают диссипацией разного знака и обобщают ранее рассмотренные.
\end{abstract}

Ключевые слова: динамическая система, интегрируемость, диссипация, трансцендентный первый интеграл.

DOI: https://doi.org/10.31857/S0869-56524896592-598

Задача определения и описания диссипации в динамической системе является довольно затруднительной. Но это может быть сделано следующим образом: вполне определённые коэффициенты в уравнениях указывают на рассеяние энергии в одних областях фазового пространства и на подкачку энергии в других. Это приводит к потере известных первых интегралов (законов сохранения), глобально выражающихся через гладкие функции.

Известным препятствием к наличию полного набора гладких первых интегралов в системе могут быть притягивающие или отталкивающие предельные множества. При их обнаружении необходимо забыть о полном наборе даже непрерывных во всём фазовом пространстве автономных первых интегралов [1].

В ряде случаев в динамике систем с диссипацией (разного знака) если и удаётся найти полный набор первых интегралов, то среди них обязательно будут первые интегралы, являющиеся трансцендентными (в смысле комплексного анализа) функциями (имеющими существенно особые точки). Поэтому результаты, полученные в данной работе, особенно важны в смысле присутствия в системе именно неконсервативного поля сил.

В ряде работ автора уже затрагивалась данная тематика (см., например, [2, 3]). В данной работе показана интегрируемость некоторых классов однородных по части переменных динамических систем девятого порядка, в которых выделяется сис-

Московский государственный университет им. М.В. Ломоносова

E-mail: shamolin@imec.msu.ru тема на касательном расслоении к четырёхмерным многообразиям. При этом силовые поля обладают диссипацией разного знака и обобщают ранее рассмотренные.

\section{1. ЗАМЕЧАНИЯ К СИСТЕМАМ МАЛЫХ НЕЧЁТНЫХ ПОРЯДКОВ}

Пусть $v, \alpha, z-$ фазовые переменные в системе, правые части которой - однородные полиномы степени 2 по переменным $v, z$ (для уравнений на $v^{*}$, $z^{*}, v \alpha^{*}$ см. также [4]). Тогда, выбирая в качестве нового времени переменную $q\left(d q=v d t, \frac{d}{d q}=\left\langle{ }^{\prime}\right\rangle, v \neq 0\right)$, ранее изучалась система третьего порядка

$$
\begin{array}{cc}
v^{\prime}=\Psi(\alpha, Z) v, & \alpha^{\prime}=-Z+b Z^{2} \delta(\alpha), \\
Z^{\prime}=-Z \Psi(\alpha, Z), & \Psi(\alpha, Z)=-b Z^{2} \frac{d \delta(\alpha)}{d \alpha},
\end{array}
$$

$z=Z v, b \geq 0, \delta(\alpha)-$ некоторая гладкая функция как система при отсутствии внешнего поля сил. При этом уравнение на $v$ отделяется, что позволяет рассматривать два оставшихся уравнения в качестве системы с одной степенью свободы на двумерном многообразии $N^{2}\{Z ; \alpha\}$ [5].

Система (1) имеет два гладких первых интеграла. Независимая подсистема на $N^{2}\{Z ; \alpha\}$ имеет рациональный по $Z$ первый интеграл (см. также $[4,6]$ ). Добавляя в систему (1) внешнее силовое поле $F(\alpha)$ при $b>0$ :

$$
\begin{gathered}
v^{\prime}=\Psi(\alpha, Z) v, \quad \alpha^{\prime}=-Z+b Z^{2} \delta(\alpha), \\
Z^{\prime}=F(\alpha)-Z \Psi(\alpha, Z),
\end{gathered}
$$

создаётся впечатление, что система осталась консервативной (что и имеет место при $b=0[2,3]$ ). 
Действительно при некотором условии у неё существует гладкий первый интеграл, структура которого напоминает интеграл полной энергии. Но дополнительного гладкого первого интеграла система, вообще говоря, не имеет. Более того, если $F(\alpha)=\delta(\alpha) \frac{d \delta(\alpha)}{d \alpha}$, дополнительный первый интеграл является трансцендентной функцией фазовых переменных (т.е. имеет существенно особые точки, означающие наличие в системе притягивающих предельных множеств) [7].

Аналогично рассматривались системы пятого и даже седьмого порядков на многообразии с ненулевыми коэффициентами связности $\Gamma_{j k}^{i}$ :

$$
\begin{aligned}
& v^{\prime}=\Psi(\alpha, Z) v, \quad \Psi(\alpha, Z)=-b\left(Z_{1}^{2}+Z_{2}^{2}+Z_{3}^{2}\right) \frac{d \delta(\alpha)}{d \alpha}, \\
& \alpha^{\prime}=-Z_{3}+b\left(Z_{1}^{2}+Z_{2}^{2}+Z_{3}^{2}\right) \delta(\alpha), \\
& Z_{3}^{\prime}=\Gamma_{3}(\alpha) f^{2}(\alpha) Z_{2}^{2}+\Gamma_{3}(\alpha) f^{2}(\alpha) Z_{1}^{2}-Z_{3} \Psi(\alpha, Z), \\
& Z_{2}^{\prime}=\left[2 \Gamma_{1}(\alpha)+\frac{d \ln |f(\alpha)|}{d \alpha}\right] Z_{2} Z_{3}- \\
& -\Gamma_{22}^{1}(\alpha, \beta) f(\alpha) g^{2}\left(\beta_{1}\right) Z_{1}^{2}-Z_{2} \Psi(\alpha, Z) \text {, } \\
& Z_{1}^{\prime}=\left[2 \Gamma_{1}(\alpha)+\frac{d \ln |f(\alpha)|}{d \alpha}\right] Z_{1} Z_{3}- \\
& -\left[2 \Gamma_{2}\left(\beta_{1}\right)+\frac{d \ln \left|g\left(\beta_{1}\right)\right|}{d \beta_{1}}\right] f(\alpha) Z_{1} Z_{2}-Z_{1} \Psi(\alpha, Z), \\
& \beta_{1}^{\prime}=Z_{2} f(\alpha), \quad \beta_{2}^{\prime}=Z_{1} f(\alpha) g\left(\beta_{1}\right),
\end{aligned}
$$

$Z=\left(Z_{1}, Z_{2}, Z_{3}\right), z_{k}=Z_{k} v, k=1,2,3, b \geq 0, \delta(\alpha), f(\alpha)$, $g\left(\beta_{1}\right)$ - некоторые гладкие функции как системы при отсутствии внешнего поля сил, в которых также присутствуют коэффициенты при параметре $b \geq 0$. Но, как и в системе (1), данные коэффициенты не нарушают консервативности, поскольку данные системы обладают полным набором (пятью) гладких первых интегралов [2, 3].

\section{2. СИСТЕМЫ ДЕВЯТОГО ПОРЯДКА ПРИ ОТСУТСТВИИ ВНЕШНЕГО СИЛОВОГО ПОЛЯ}

Пусть $v, \alpha, \beta=\left(\beta_{1}, \beta_{2}, \beta_{3}\right), z=\left(z_{1}, \ldots, z_{4}\right)-$ фазовые переменные в системе, правые части которой однородные полиномы степени 2 по переменным $v, z$ (для уравнений на $v^{*}, z^{*}, v \alpha, v \beta^{*}$ ), с коэффициентами, зависящими от $\alpha, \beta$. Тогда, выбирая в качестве нового времени величину $q\left(d q=v d t, \frac{d}{d q}=\left\langle{ }^{\prime}\right\rangle\right.$, $v \neq 0$ ), будем рассматривать систему девятого порядка

$$
v^{\prime}=\Psi(\alpha, Z) v
$$

$$
\begin{aligned}
& \Psi(\alpha, Z)=-b\left(Z_{1}^{2}+\ldots+Z_{4}^{2}\right) \frac{d \delta(\alpha)}{d \alpha} ; \\
& \alpha^{\prime}=-Z_{4}+b\left(Z_{1}^{2}+\ldots+Z_{4}^{2}\right) \delta(\alpha), \\
& Z_{4}^{\prime}=\Gamma_{11}^{\alpha}(\alpha, \beta) f_{1}^{2}(\alpha) Z_{3}^{2}+ \\
& +\Gamma_{22}^{\alpha}(\alpha, \beta) f_{2}^{2}(\alpha) g_{1}^{2}\left(\beta_{1}\right) Z_{2}^{2}+ \\
& +\Gamma_{33}^{\alpha}(\alpha, \beta) f_{3}^{2}(\alpha) g_{2}^{2}\left(\beta_{1}\right) h^{2}\left(\beta_{2}\right) Z_{1}^{2}- \\
& -Z_{4} \Psi(\alpha, Z) \text {, } \\
& Z_{3}^{\prime}=\left[2 \Gamma_{\alpha 1}^{1}(\alpha, \beta)+\frac{d \ln \left|f_{1}(\alpha)\right|}{d \alpha}\right] Z_{3} Z_{4}- \\
& -\Gamma_{22}^{1}(\alpha, \beta) \frac{f_{2}^{2}(\alpha)}{f_{1}(\alpha)} g_{1}^{2}\left(\beta_{1}\right) Z_{2}^{2}- \\
& -\Gamma_{33}^{1}(\alpha, \beta) \frac{f_{3}^{2}(\alpha)}{f_{1}(\alpha)} g_{2}^{2}\left(\beta_{1}\right) h^{2}\left(\beta_{2}\right) Z_{1}^{2}-Z_{3} \Psi(\alpha, Z), \\
& Z_{2}^{\prime}=\left[2 \Gamma_{\alpha 2}^{2}(\alpha, \beta)+\frac{d \ln \left|f_{2}(\alpha)\right|}{d \alpha}\right] Z_{2} Z_{4}- \\
& -\left[2 \Gamma_{12}^{2}(\alpha, \beta)+\frac{d \ln \left|g_{1}\left(\beta_{1}\right)\right|}{d \beta_{1}}\right] f_{1}(\alpha) Z_{2} Z_{3}- \\
& -\Gamma_{33}^{2}(\alpha, \beta) \frac{f_{3}^{2}(\alpha)}{f_{2}(\alpha)} \frac{g_{2}^{2}\left(\beta_{1}\right)}{g_{1}\left(\beta_{1}\right)} h^{2}\left(\beta_{2}\right) Z_{1}^{2}-Z_{2} \Psi(\alpha, Z), \\
& Z_{1}^{\prime}=\left[2 \Gamma_{\alpha 3}^{3}(\alpha, \beta)+\frac{d \ln \left|f_{3}(\alpha)\right|}{d \alpha}\right] Z_{1} Z_{4}- \\
& -\left[2 \Gamma_{13}^{3}(\alpha, \beta)+\frac{d \ln \left|g_{2}\left(\beta_{1}\right)\right|}{d \beta_{1}}\right] f_{1}(\alpha) Z_{1} Z_{3}- \\
& -\left[2 \Gamma_{23}^{3}(\alpha, \beta)+\frac{d \ln \left|h\left(\beta_{2}\right)\right|}{d \beta_{2}}\right] f_{2}(\alpha) g_{1}\left(\beta_{1}\right) Z_{1} Z_{2}- \\
& -Z_{1} \Psi(\alpha, Z), \\
& \beta_{1}^{\prime}=Z_{3} f_{1}(\alpha), \quad \beta_{2}^{\prime}=Z_{2} f_{2}(\alpha) g_{1}\left(\beta_{1}\right), \\
& \beta_{3}^{\prime}=Z_{1} f_{3}(\alpha) g_{2}\left(\beta_{1}\right) h\left(\beta_{2}\right),
\end{aligned}
$$

$Z=\left(Z_{1}, \ldots, Z_{4}\right), Z_{q}=Z_{q} v, q=1, \ldots, 4, b \geq 0, \delta(\alpha), f_{k}(\alpha)$, $k=1,2,3, g_{1}\left(\beta_{1}\right), l=1,2, h\left(\beta_{2}\right)-$ некоторые гладкие функции как системы при отсутствии внешнего поля сил. При этом уравнение (2) отделяется, что даёт возможность рассматривать уравнения (3) в качестве независимой системы (с четырьмя степенями свободы) на восьмимерном многообразии $N^{8}\left\{Z_{4}, \ldots, Z_{1}\right.$; $\left.\alpha, \beta_{1}, \beta_{2}, \beta_{3}\right\}=T M^{4}\left\{Z_{4}, \ldots, Z_{1} ; \alpha, \beta_{1}, \beta_{2}, \beta_{3}\right\}$ (касательном расслоении гладкого четырёхмерного многообразия $M^{4}\left\{\alpha, \beta_{1}, \beta_{2}, \beta_{3}\right\}$, см. также [2,7]).

Рассмотрим структуру системы (3). Она для простоты соответствует следующим уравнениям геодезических линий на касательном расслоении $T M^{4}\left\{\alpha^{\cdot}, \beta_{1}^{\cdot}, \beta_{2}^{\cdot}, \beta_{3}^{\cdot} ; \alpha, \beta_{1}, \beta_{2}, \beta_{3}\right\}$ многообразия $M^{4}\left\{\alpha, \beta_{1}, \beta_{2}, \beta_{3}\right\}[8]$ (в частности, сферы или более общих поверхностей вращения - с 12 ненулевыми коэффициентами связности): 
$\alpha^{*}+\Gamma_{11}^{\alpha}(\alpha, \beta) \beta_{1}^{\cdot 2}+\Gamma_{22}^{\alpha}(\alpha, \beta) \beta_{2}^{\cdot 2}+\Gamma_{33}^{\alpha}(\alpha, \beta) \beta_{3}^{\cdot 2}=0$, $\beta_{1}^{\ddot{*}}+2 \Gamma_{\alpha 1}^{1}(\alpha, \beta) \alpha \beta_{1}^{\bullet}+\Gamma_{22}^{1}(\alpha, \beta) \beta_{2}^{\cdot 2}+\Gamma_{33}^{1}(\alpha, \beta) \beta_{3}^{\cdot 2}=0$,

$$
\begin{gathered}
\beta_{2}^{\ddot{2}}+2 \Gamma_{\alpha 2}^{2}(\alpha, \beta) \alpha \beta_{2}^{\cdot}+2 \Gamma_{12}^{2}(\alpha, \beta) \beta_{1}^{*} \beta_{2}^{\cdot}+ \\
+\Gamma_{33}^{2}(\alpha, \beta) \beta_{3}^{\cdot 2}=0, \\
\beta_{3}^{\ddot{2}}+2 \Gamma_{\alpha 3}^{3}(\alpha, \beta) \alpha \beta_{3}^{\cdot}+2 \Gamma_{13}^{3}(\alpha, \beta) \beta_{1}^{*} \beta_{3}^{\cdot}+ \\
+2 \Gamma_{23}^{3}(\alpha, \beta) \beta_{2}^{\cdot} \beta_{3}^{\cdot}=0 .
\end{gathered}
$$

Действительно, выбрав новые координаты $Z_{1}, \ldots$ ..., $Z_{4}$ в касательном пространстве

$$
\alpha^{\prime}=-Z_{4}, \quad \beta_{1}^{\prime}=Z_{3} f_{1}(\alpha),
$$

$\beta_{2}^{\prime}=Z_{2} f_{2}(\alpha) g_{1}\left(\beta_{1}\right), \quad \beta_{3}^{\prime}=Z_{1} f_{3}(\alpha) g_{2}\left(\beta_{1}\right) h\left(\beta_{2}\right)$,

мы получаем соотношения на них в следующем виде (ср. с системой (3)):

$$
\begin{gathered}
Z_{1}^{\prime}=\left[2 \Gamma_{\alpha 3}^{3}(\alpha, \beta)+\frac{d \ln \left|f_{3}(\alpha)\right|}{d \alpha}\right] Z_{1} Z_{4}- \\
-\left[2 \Gamma_{13}^{3}(\alpha, \beta)+\frac{d \ln \left|g_{2}\left(\beta_{1}\right)\right|}{d \beta_{1}}\right] f_{1}(\alpha) Z_{1} Z_{3}- \\
-\left[2 \Gamma_{23}^{3}(\alpha, \beta)+\frac{d \ln \left|h\left(\beta_{2}\right)\right|}{d \beta_{2}}\right] f_{2}(\alpha) g_{1}\left(\beta_{1}\right) Z_{1} Z_{2}, \\
Z_{2}^{\prime}=\left[2 \Gamma_{\alpha 2}^{2}(\alpha, \beta)+\frac{\left.d \ln \left|f_{2}(\alpha)\right|\right]}{d \alpha}\right] Z_{2} Z_{4}- \\
-\left[2 \Gamma_{12}^{2}(\alpha, \beta)+\frac{d \ln \left|g_{1}\left(\beta_{1}\right)\right|}{d \beta_{1}}\right] f_{1}(\alpha) Z_{2} Z_{3}- \\
-\Gamma_{33}^{2}(\alpha, \beta) \frac{f_{3}^{2}(\alpha) \frac{g_{2}^{2}\left(\beta_{1}\right)}{f_{2}(\alpha)} h_{1}^{2}\left(\beta_{2}\right) Z_{1}^{2},}{\left.g_{1}\right)} \\
Z_{3}^{\prime}=\left[2 \Gamma_{\alpha 1}^{1}(\alpha, \beta)+\frac{d \ln \left|f_{1}(\alpha)\right|}{d \alpha}\right] Z_{3} Z_{4}- \\
-\Gamma_{22}^{1}(\alpha, \beta) \frac{f_{2}^{2}(\alpha)}{f_{1}(\alpha)} g_{1}^{2}\left(\beta_{1}\right) Z_{2}^{2}- \\
-\Gamma_{33}^{1}(\alpha, \beta) \frac{f_{3}^{2}(\alpha)}{f_{1}(\alpha)} g_{2}^{2}\left(\beta_{1}\right) h^{2}\left(\beta_{2}\right) Z_{1}^{2}, \\
Z_{4}^{\prime}=\Gamma_{11}^{\alpha}(\alpha, \beta) f_{1}^{2}(\alpha) Z_{3}^{2}+ \\
+\Gamma_{22}^{\alpha}(\alpha, \beta) f_{2}^{2}(\alpha) g_{1}^{2}\left(\beta_{1}\right) Z_{2}^{2}+ \\
+\Gamma_{33}^{\alpha}(\alpha, \beta) f_{3}^{2}(\alpha) g_{2}^{2}\left(\beta_{1}\right) h^{2}\left(\beta_{2}\right) Z_{1}^{2},
\end{gathered}
$$

и система (4) почти всюду эквивалентна (если отождествить независимые переменные) совокупности (5), (6), которая прежде всего присутствует в (3).

Далее в системе (3) также присутствуют коэффициенты при параметре $b \geq 0$. Но, как и в системе (1), они не нарушают консервативности, поскольку система (2), (3) при некоторых естественных условиях обладает полным набором (шестью) гладких первых интегралов.
Пре дл о жен и е 1. Если всюду на своей области определения справедлива система равенств

$$
\begin{aligned}
& 2 \Gamma_{\alpha 1}^{1}(\alpha, \beta)+\frac{d \ln \left|f_{1}(\alpha)\right|}{d \alpha}+\Gamma_{11}^{\alpha}(\alpha, \beta) f_{1}^{2}(\alpha) \equiv 0, \\
& 2 \Gamma_{\alpha 2}^{2}(\alpha, \beta)+\frac{d \ln \left|f_{2}(\alpha)\right|}{d \alpha}+\Gamma_{22}^{\alpha}(\alpha, \beta) f_{2}^{2}(\alpha) g_{1}^{2}\left(\beta_{1}\right) \equiv 0, \\
& {\left[2 \Gamma_{12}^{2}(\alpha, \beta)+\frac{d \ln \left|g_{1}\left(\beta_{1}\right)\right|}{d \beta_{1}}\right] f_{1}^{2}(\alpha)+} \\
& +\Gamma_{22}^{1}(\alpha, \beta) f_{2}^{2}(\alpha) g_{1}^{2}\left(\beta_{1}\right) \equiv 0, \\
& 2 \Gamma_{\alpha 3}^{3}(\alpha, \beta)+\frac{d \ln \left|f_{3}(\alpha)\right|}{d \alpha}+ \\
& +\Gamma_{33}^{\alpha}(\alpha, \beta) f_{3}^{2}(\alpha) g_{2}^{2}\left(\beta_{1}\right) h^{2}\left(\beta_{2}\right) \equiv 0, \\
& {\left[2 \Gamma_{13}^{3}(\alpha, \beta)+\frac{d \ln \left|g_{2}\left(\beta_{1}\right)\right|}{d \beta_{1}}\right] f_{1}^{2}(\alpha)+} \\
& +\Gamma_{33}^{1}(\alpha, \beta) f_{3}^{2}(\alpha) g_{2}^{2}\left(\beta_{1}\right) h^{2}\left(\beta_{2}\right) \equiv 0, \\
& {\left[2 \Gamma_{23}^{3}(\alpha, \beta)+\frac{d \ln \left|h\left(\beta_{2}\right)\right|}{d \beta_{2}}\right] f_{2}^{2}(\alpha) g_{1}^{2}\left(\beta_{1}\right)+} \\
& +\Gamma_{33}^{2}(\alpha, \beta) f_{3}^{2}(\alpha) g_{2}^{2}\left(\beta_{1}\right) h^{2}\left(\beta_{2}\right) \equiv 0,
\end{aligned}
$$

то система (2), (3) имеет аналитический первый интеграл вида

$\Phi_{1}\left(v ; Z_{4}, \ldots, Z_{1}\right)=v^{2}\left(Z_{1}^{2}+\ldots+Z_{4}^{2}\right)=C_{1}^{2}=$ const. (8)

Можно доказать отдельную теорему существования решения $f_{k}(\alpha), k=1,2,3, g_{l}\left(\beta_{1}\right), l=1,2, h\left(\beta_{2}\right)$ системы (7) для условий наличия аналитического интеграла (8) для системы (5), (6) уравнений геодезических. Но в дальнейшем при изучении динамических систем с диссипацией вся группа условий (7) нам не потребуется. Тем не менее в дальнейшем будем предполагать в уравнениях (5) выполнение равенств

$$
f_{1}(\alpha) \equiv f_{2}(\alpha) \equiv f_{3}(\alpha)=f(\alpha),
$$

при этом функции $g_{l}\left(\beta_{1}\right), l=1,2, h\left(\beta_{2}\right)$ должны удовлетворять преобразованным уравнениям из (7):

$$
\begin{aligned}
& 2 \Gamma_{12}^{2}(\alpha, \beta)+\frac{d \ln \left|g_{1}\left(\beta_{1}\right)\right|}{d \beta_{1}}+\Gamma_{22}^{1}(\alpha, \beta) g_{1}^{2}\left(\beta_{1}\right) \equiv 0, \\
& 2 \Gamma_{13}^{3}(\alpha, \beta)+\frac{d \ln \left|g_{2}\left(\beta_{1}\right)\right|}{d \beta_{1}}+ \\
& +\Gamma_{33}^{1}(\alpha, \beta) g_{2}^{2}\left(\beta_{1}\right) h^{2}\left(\beta_{2}\right) \equiv 0, \\
& {\left[2 \Gamma_{23}^{3}(\alpha, \beta)+\frac{d \ln \left|h\left(\beta_{2}\right)\right|}{d \beta_{2}}\right] g_{1}^{2}\left(\beta_{1}\right)+} \\
& +\Gamma_{33}^{2}(\alpha, \beta) g_{2}^{2}\left(\beta_{1}\right) h^{2}\left(\beta_{2}\right) \equiv 0 .
\end{aligned}
$$

Таким образом, функции $g_{l}\left(\beta_{1}\right), l=1,2, h\left(\beta_{2}\right)$ пока зависят от коэффициентов связности, а ограничения на функцию $f(\alpha)$ будут даны ниже. 
Предложен ие 2. Если выполнены свойства (9), (10), при этом справедливы равенства

$$
\Gamma_{\alpha 1}^{1}(\alpha, \beta) \equiv \Gamma_{\alpha 2}^{2}(\alpha, \beta) \equiv \Gamma_{\alpha 3}^{3}(\alpha, \beta)=\Gamma_{1}(\alpha),
$$

то система (2), (3) имеет гладкий первый интеграл следующего вида:

$$
\begin{gathered}
\Phi_{2}\left(v ; Z_{3}, Z_{2}, Z_{1} ; \alpha\right)= \\
=v^{2} \sqrt{Z_{1}^{2}+Z_{2}^{2}+Z_{3}^{3}} \delta(\alpha)=C_{2}=\mathrm{const},
\end{gathered}
$$

при этом функция $\delta(\alpha)$ должна удовлетворять равенству

$$
\delta(\alpha)=A_{1} f(\alpha) \exp \left\{2 \int_{\alpha_{0}}^{\alpha} \Gamma_{1}(b) d b\right\}, \quad A_{1}=\text { const } .
$$

Предложение 3. Если выполнены условия предложения 2, а также

$$
g_{1}\left(\beta_{1}\right) \equiv g_{2}\left(\beta_{1}\right)=g\left(\beta_{1}\right),
$$

при этом справедливы равенства

$$
\Gamma_{12}^{2}(\alpha, \beta) \equiv \Gamma_{13}^{3}(\alpha, \beta)=\Gamma_{2}\left(\beta_{1}\right),
$$

то система (2), (3) имеет гладкий первый интеграл следующего вида:

$$
\begin{gathered}
\Phi_{3}\left(v ; Z_{2}, Z_{1} ; \alpha, \beta_{1}\right)= \\
=v^{2} \sqrt{Z_{1}^{2}+Z_{2}^{2}} \delta(\alpha) \Psi_{1}\left(\beta_{1}\right)=C_{3}=\mathrm{const}, \\
\Psi_{1}\left(\beta_{1}\right)=g\left(\beta_{1}\right) \exp \left\{2 \int_{\beta_{10}}^{\beta_{1}} \Gamma_{2}(b) d b\right\} .
\end{gathered}
$$

Предл ожен ие 4. Если выполнены условия предложений 2, 3, при этом справедливо равенство

$$
\Gamma_{23}^{3}(\alpha, \beta)=\Gamma_{3}\left(\beta_{2}\right),
$$

то система (2), (3) имеет гладкий первый интеграл следующего вида:

$$
\begin{gathered}
\Phi_{4}\left(v ; Z_{1} ; \alpha, \beta_{1}, \beta_{2}\right)= \\
=v^{2} Z_{1} \delta(\alpha) \Psi_{1}\left(\beta_{1}\right) \Psi_{2}\left(\beta_{2}\right)=C_{4}=\text { const } \\
\Psi_{2}\left(\beta_{2}\right)=h\left(\beta_{2}\right) \exp \left\{2 \int_{\beta_{20}}^{\beta_{2}} \Gamma_{3}(b) d b\right\} .
\end{gathered}
$$

Предложение 5. Пусть выполнены свойства (9), (14) $и$

$$
\begin{aligned}
& \Gamma_{11}^{\alpha}(\alpha, \beta) \equiv \Gamma_{22}^{\alpha}(\alpha, \beta) g^{2}\left(\beta_{1}\right) \equiv \\
\equiv & \Gamma_{33}^{\alpha}(\alpha, \beta) g^{2}\left(\beta_{1}\right) h^{2}\left(\beta_{2}\right)=\Gamma_{4}(\alpha) .
\end{aligned}
$$

Тогда система (2), (3) имеет гладкий первый интеграл следующего вида:

$$
\Phi_{0}\left(v ; Z_{4} ; \alpha\right)=v^{2}\left(1-2 b Z_{4} \delta(\alpha)\right)=C_{0}=\text { const, }(20)
$$

если функция $\delta(\alpha)$ удовлетворяет равенству

$$
\delta(\alpha)=A_{2} \exp \left\{-\int_{\alpha_{0}}^{\alpha} \Gamma_{4}(b) f^{2}(b) d b\right\}, \quad A_{2}=\text { const. }
$$

В частности, если выполнены свойства (7), (9), (11), (13), (14), (19), то система (2), (3) имеет гладкий первый интеграл вида (20).

Предложение 6. Если выполнены условия предложений 3, 4, то система (2), (3) имеет гладкий первый интеграл следующего вида:

$$
\begin{gathered}
\Phi_{5}\left(\beta_{2}, \beta_{3}, C_{3}, C_{4}\right)= \\
=\beta_{3}+\int_{\beta_{20}}^{\beta_{2}} \frac{C_{4} h(b)}{\sqrt{C_{3}^{2} \Psi_{2}^{2}(b)-C_{4}^{2}}} d b=C_{5}=\text { const },
\end{gathered}
$$

где после взятия интеграла (21) вместо постоянных $\mathrm{C}_{3}, \mathrm{C}_{4}$ можно подставить левые части равенств (16), (18) соответственно.

Те о рема 1. Если выполнены условия предложений 1-6, то система (2), (3) обладает полным набором (шестью) гладких независимых первых интегралов вида (8), (12), (16), (18), (20), (21).

\section{3. ВВЕДЕНИЕ ВНЕШНЕГО СИЛОВОГО ПОЛЯ}

Модифицируем систему (2), (3) при условиях (9), (11), (14), (15), (17), (19) при наличии двух ключевых параметров $b, b_{1} \geq 0$, введя внешнее силовое поле. Если ввести такое поле, добавив коэффициент $F(\alpha)$ лишь в уравнение на $Z_{4}^{\prime}$ системы (22), (23) и даже положив при этом $b_{1}=0$, то полученная система, вообще говоря, не будет консервативной. Консервативность будет при дополнительном условии $b=0$. Но мы расширим введение силового поля, положив $b_{1}>0$. Рассматриваемая система на прямом произведении числового луча и касательного расслоения $T M^{4}\left\{Z_{4}, \ldots, Z_{1} ; \alpha, \beta_{1}, \beta_{2}, \beta_{3}\right\}$ примет вид

$$
v^{\prime}=\Psi(\alpha, \beta),
$$

$\Psi(\alpha, Z)=-b\left(Z_{1}^{2}+\ldots+Z_{4}^{2}\right) \frac{d \delta(\alpha)}{d \alpha}+b_{1} F(\alpha) \delta(\alpha) ;$

$$
\begin{gathered}
\alpha^{\prime}=-Z_{4}+b\left(Z_{1}^{2}+\ldots+Z_{4}^{2}\right) \delta(\alpha)+b_{1} F(\alpha) \tilde{f}(\alpha), \\
\tilde{f}(\alpha)=\frac{\mu-\delta^{2}(\alpha)}{d \delta(\alpha) / d \alpha}, \\
Z_{4}^{\prime}=F(\alpha)+\Gamma_{4}(\alpha) f^{2}(\alpha) Z_{3}^{2}+\Gamma_{4}(\alpha) f^{2}(\alpha) Z_{2}^{2}+ \\
+\Gamma_{4}(\alpha) f^{2}(\alpha) Z_{1}^{2}-Z_{4} \Psi(\alpha, Z), \\
Z_{3}^{\prime}=\left[2 \Gamma_{1}(\alpha)+\frac{d \ln |f(\alpha)|}{d \alpha}\right] Z_{3} Z_{4}- \\
-\Gamma_{22}^{1}(\alpha, \beta) f(\alpha) g^{2}\left(\beta_{1}\right) Z_{2}^{2}- \\
-\Gamma_{33}^{1}(\alpha, \beta) f(\alpha) g^{2}\left(\beta_{1}\right) h^{2}\left(\beta_{2}\right) Z_{1}^{2}-Z_{3} \Psi(\alpha, Z),
\end{gathered}
$$




$$
\begin{gathered}
Z_{2}^{\prime}=\left[2 \Gamma_{1}(\alpha)+\frac{d \ln |f(\alpha)|}{d \alpha}\right] Z_{2} Z_{4}- \\
-\left[2 \Gamma_{2}\left(\beta_{1}\right)+\frac{d \ln \left|g\left(\beta_{1}\right)\right|}{d \beta_{1}}\right] f(\alpha) Z_{2} Z_{3}- \\
-\Gamma_{33}^{2}(\alpha, \beta) f(\alpha) g\left(\beta_{1}\right) h^{2}\left(\beta_{2}\right) Z_{1}^{2}-Z_{2} \Psi(\alpha, Z), \\
Z_{1}^{\prime}=\left[2 \Gamma_{1}(\alpha)+\frac{d \ln |f(\alpha)|}{d \alpha}\right] Z_{1} Z_{4}- \\
-\left[2 \Gamma_{2}\left(\beta_{1}\right)+\frac{d \ln \left|g\left(\beta_{1}\right)\right|}{d \beta_{1}}\right] f(\alpha) Z_{1} Z_{3}- \\
-\left[2 \Gamma_{3}\left(\beta_{2}\right)+\frac{d \ln \left|h\left(\beta_{2}\right)\right|}{d \beta_{2}}\right] f(\alpha) g\left(\beta_{1}\right) Z_{1} Z_{2}- \\
-Z_{1} \Psi(\alpha, Z), \\
\beta_{1}^{\prime}=Z_{3} f(\alpha), \beta_{2}^{\prime}=Z_{2} f(\alpha) g\left(\beta_{1}\right), \\
\beta_{3}^{\prime}=Z_{1} f(\alpha) g\left(\beta_{1}\right) h\left(\beta_{2}\right),
\end{gathered}
$$

$\mu=$ const. При этом коэффициенты консервативной составляюшей силового поля содержат параметр $b$, а неконсервативной составляющей внешнего поля параметр $b_{1}$.

Силовое поле в уравнениях на $v^{\prime}, Z_{1}^{\prime}, \ldots, Z_{4}^{\prime}$ определяется функцией $\Psi(\alpha, Z)$. Опишем введение силового поля в виде двумерного столбца, в первой строке которого стоят коэффициенты из функции $\Psi(\alpha, Z)$, а во второй - коэффициенты из уравнения на $\alpha^{\prime}$. Таким образом, совместное силовое поле (в котором присутствуют три параметра $\left.b, b_{1} \geq 0, \mu\right)$ будут иметь вид

$$
U\left(\begin{array}{c}
b\left(Z_{1}^{2}+\ldots+Z_{4}^{2}\right) \\
b_{1} F(\alpha)
\end{array}\right), \quad U=\left(\begin{array}{rr}
-\frac{d \delta(\alpha)}{d \alpha} & \delta(\alpha) \\
\delta(\alpha) & \tilde{f}(\alpha)
\end{array}\right),
$$

где $U$ - преобразование с определителем, равным - $\mu$, являющееся унимодулярным преобразованием при $\mu= \pm 1$. Такое преобразование вносит в систему диссипацию (как одного знака, так и другого, см. также $[2,3])$.

\section{4. ИНТЕГРИРОВАНИЕ СИСТЕМЫ С ДИССИПАЦИЕЙ}

Перейдём теперь к интегрированию системы девятого порядка (22), (23) при выполнении свойств (10). Она также допускает отделение независимой подсистемы седьмого порядка.

Введём также (по аналогии с (10)) ограничение и на функцию $f(\alpha)$ : она должна удовлетворять преобразованному первому равенству из (7):

$$
2 \Gamma_{1}(\alpha)+\frac{d \ln |f(\alpha)|}{d \alpha}+\Gamma_{4}(\alpha) f^{2}(\alpha) \equiv 0
$$

Для полного интегрирования системы (23) необходимо знать, вообще говоря, семь независимых первых интегралов. Однако после замены переменных

$$
\begin{gathered}
w_{4}=Z_{4}, \quad w_{3}=\sqrt{Z_{1}^{2}+Z_{2}^{2}+Z_{3}^{2}}, \\
w_{2}=\frac{Z_{2}}{Z_{1}}, \quad w_{1}=\frac{Z_{3}}{\sqrt{Z_{1}^{2}+Z_{2}^{2}}}
\end{gathered}
$$

система (23) распадается следующим образом:

$$
\begin{gathered}
\alpha^{\prime}=-w_{4}+b\left(w_{4}^{2}+w_{3}^{2}\right) \delta(\alpha)+b_{1} F(\alpha) \tilde{f}(\alpha), \\
w_{4}^{\prime}=F(\alpha)+\Gamma_{4}(\alpha) f^{2}(\alpha) w_{3}^{2}-w_{4} \Psi(\alpha, w), \\
w_{3}^{\prime}=\left[2 \Gamma_{1}(\alpha)+\frac{d \ln |f(\alpha)|]}{d \alpha}\right] w_{3} w_{4}-w_{3} \Psi(\alpha, w) ; \\
w_{2}^{\prime}= \pm w_{3} \sqrt{1+w_{2}^{2}} f(\alpha) g\left(\beta_{1}\right)\left[2 \Gamma_{3}\left(\beta_{2}\right)+\frac{d \ln \left|h\left(\beta_{2}\right)\right|}{d \beta_{2}}\right], \\
\beta_{2}^{\prime}=( \pm) \frac{w_{2} w_{3}}{\sqrt{1+w_{2}^{2}}} f(\alpha) g\left(\beta_{1}\right) ; \\
w_{1}^{\prime}= \pm w_{3} \sqrt{1+w_{1}^{2}} f(\alpha)\left[2 \Gamma_{2}\left(\beta_{1}\right)+\frac{d \ln \left|g\left(\beta_{1}\right)\right|}{d \beta_{1}}\right], \\
\beta_{1}^{\prime}=( \pm) \frac{w_{1} w_{3}}{\sqrt{1+w_{1}^{2}}} f(\alpha) ; \\
\beta_{3}^{\prime}= \pm \frac{w_{3}}{\sqrt{1+w_{2}^{2}}} f(\alpha) g\left(\beta_{1}\right) h\left(\beta_{2}\right), \\
\Psi(\alpha, w)=-b\left(w_{4}^{2}+w_{3}^{2}\right) \frac{d \delta(\alpha)}{d \alpha}+b_{1} F(\alpha) \delta(\alpha) .
\end{gathered}
$$

Видно, что для полной интегрируемости системы (23) достаточно указать два независимых первых интеграла системы (25), по одному - для систем (26) и (27) (после соответствующих замен независимых переменных) и дополнительный первый интеграл, “привязывающий” уравнение (28) (т.е. всего пять).

Теорема 2. Пусть для некоторых $\kappa, \lambda \in \mathbf{R}$ выполняются равенства

$$
\begin{gathered}
\Gamma_{4}(\alpha) f^{2}(\alpha)=\kappa \frac{d}{d \alpha} \ln \mid \delta(\alpha), \\
F(\alpha)=\lambda \frac{d}{d \alpha} \frac{\delta^{2}(\alpha)}{2} .
\end{gathered}
$$

Тогда система (22), (23) при выполнении свойств (10), (24) обладает шестью независимыми (вообще говоря, трансиендентными [9] в смысле комплексного анализа) первыми интегралами.

Первое равенство из (29) можно назвать геометрическим, а второе - энергетическим.

В общем случае первые интегралы выписываются громоздко. В частности, если $\kappa=-1$, то явный вид одного из первых интегралов таков: 


$$
\begin{gathered}
\Theta_{1}\left(w_{4}, w_{3} ; \alpha\right)=G_{1}\left(\frac{w_{4}}{\delta(\alpha)}, \frac{w_{3}}{\delta(\alpha)}\right)= \\
=\frac{w_{4}^{2}+w_{3}^{2}-b_{1} \lambda \mu w_{4} \delta(\alpha)+\lambda \delta^{2}(\alpha)}{w_{3} \delta(\alpha)}=C_{1}=\text { const. }
\end{gathered}
$$

При помощи интеграла (30) получается и дополнительный первый интеграл для системы (23), который имеет следующий структурный вид:

$$
\begin{aligned}
\Theta_{2}\left(w_{4}, w_{3} ; \alpha\right) & =G_{2}\left(\delta(\alpha), \frac{w_{4}}{\delta(\alpha)}, \frac{w_{3}}{\delta(\alpha)}\right)= \\
& =C_{2}=\text { const. }
\end{aligned}
$$

Выражение первого интеграла (31) через конечную комбинацию элементарных функций зависит не только от вычисления квадратур, но также и от явного вида функции $\delta(\alpha)$. Например, при $\kappa=-1$ этот первый интеграл найдётся из уравнения Бернулли

$$
\begin{gathered}
\frac{d \delta}{d u_{4}}=\frac{\left(b_{1} \lambda \mu-u_{4}\right) \delta+b \delta^{3}\left(U^{2}\left(C_{1}, u_{4}\right)+u_{4}^{2}\right)-b_{1} \lambda \delta^{3}}{\lambda-b_{1} \lambda \mu u_{4}+u_{4}^{2}-U^{2}\left(C_{1}, u_{4}\right)}, \\
U\left(C_{1}, u_{4}\right)=\frac{C_{1} \pm \sqrt{C_{1}^{2}-4\left(\lambda-b_{1} \lambda \mu u_{4}+u_{4}^{2}\right)}}{2}, \\
u_{4}=\frac{w_{4}}{\delta(\alpha)} .
\end{gathered}
$$

При этом после взятия этого интеграла вместо $C_{1}$ можно подставить левую часть равенства (30).

Первые интегралы для независимых (после замен независимых переменных) подсистем (26) и (27) будут иметь вид

$\Theta_{2+s}\left(w_{s} ; \beta_{s}\right)=\frac{\sqrt{1+w_{s}^{2}}}{\Psi_{s}\left(\beta_{s}\right)}=C_{2+s}=$ const, $s=1,2$,

о функциях $\Psi_{s}\left(\beta_{s}\right)$ см. (16), (18). Дополнительный первый интеграл, “привязывающий” уравнение (28), находится по аналогии с (21):

$$
\begin{gathered}
\Theta_{5}\left(\beta_{2}, \beta_{3}, C_{3}, C_{4}\right)= \\
=\beta_{3}+\int_{\beta_{20}}^{\beta_{2}} \frac{C_{4} h(b)}{\sqrt{C_{3}^{2} \Psi_{2}^{2}(b)-C_{4}^{2}}} d b=C_{5}=\text { const },
\end{gathered}
$$

где после взятия этого интеграла вместо постоянных $C_{3}, C_{4}$ можно подставить соответствующие левые части равенств (32).

Кроме того, у системы (22), (23) существует гладкий первый интеграл (по аналогии с (20), “привязывающий” уравнение (22)), который, например, при $b=b_{1}, \mu=1$ примет вид

$$
\begin{gathered}
\Theta_{0}\left(v ; w_{4}, w_{3} ; \alpha\right)= \\
=v^{2}\left(1-2 b w_{4} \delta(\alpha)+b^{2}\left(w_{4}^{2}+w_{3}^{2}\right)\right)= \\
C_{0}=\text { const. }
\end{gathered}
$$

Справедлива и теорема, обратная к теореме 2.

Те орема 3. Условия (10), (24), (29) (например, при $\kappa=-1)$ являются необходимыми условиями существования первого интеграла (30) для системы (22), (23).

\section{5. СТРОЕНИЕ \\ ПЕРВЫХ ИНТЕГРАЛОВ \\ ДЛЯ СИСТЕМ С ДИССИПАЦИЕЙ}

Если $\alpha-$ периодическая координата периода $2 \pi$, то система (22), (23) обладает переменной диссипацией с нулевым средним [7]. При этом при $F(\alpha) \equiv 0$ она превращается в систему консервативную (2), (3). Последняя, в частности, обладает двумя гладкими первыми интегралами вида (8), (12). Более того, если функция $F(\alpha)$ не равна тождественно нулю, но $b_{1}=0$, то система (22), (23) при втором условии из (29) обладает первым интегралом вида

$$
\begin{gathered}
\Xi_{0}\left(v ; Z_{4}, \ldots, Z_{1} ; \alpha\right)= \\
=v^{2}\left(Z_{1}^{2}+\ldots+Z_{4}^{2}+\lambda \delta^{2}(\alpha)\right)=\text { const } .
\end{gathered}
$$

Очевидно, что отношение двух первых интегралов (33), (12) также является первым интегралом системы (22), (23) при неравенстве функции $F(\alpha)$ тождественно нулю, $b_{1}=0$. Но при $b_{1}>0$ каждая из функций

$$
\begin{gathered}
\Xi_{b_{1}}\left(v ; Z_{4}, \ldots, Z_{1} ; \alpha\right)= \\
=v^{2}\left(Z_{1}^{2}+\ldots+Z_{4}^{2}-b_{1} \lambda \mu Z_{4} \delta(\alpha)+\lambda \delta^{2}(\alpha)\right)
\end{gathered}
$$

и (12) по отдельности не является первым интегралом системы (22), (23). Однако отношение функций (34), (12) является первым интегралом (30) системы (22), (23) (при к $=-1$ ) при любом $b_{1}>0$.

Вообще же, как и указывалось ранее, для систем с диссипацией трансцендентность функций (в смысле наличия существенно особых точек) как первых интегралов наследуется из нахождения в системе притягивающих или отталкивающих предельных множеств (см. также [1-3]).

\section{6. НЕКОТОРЫЕ ПРИЛОЖЕНИЯ}

Выделим существенный случай для функции $f(\alpha)$, определяющей метрику на четырёхмерной сфере, и $\delta(\alpha)$ :

$$
f(\alpha)=\frac{\cos \alpha}{\sin \alpha}, \quad \delta(\alpha)=\sin \alpha .
$$

Случай (35) формирует класс систем (22), (23) при $\mu=1$, соответствующих движению пятимерного динамически симметричного твёрдого тела на нулевых уровнях циклических интегралов в неконсервативном поле сил [8]. В частности, при $\delta(\alpha) \equiv$ 
$\equiv F(\alpha) \equiv 0$ рассматриваемая система описывает геодезический поток на четырёхмерной сфере. В случае (35), если $\delta(\alpha)=\frac{F(\alpha)}{\cos \alpha}$, то система описывает движение пятимерного твёрдого тела в силовом поле $F(\alpha)$ под действием следящей силы. В частности, если $F(\alpha)=\sin \alpha \cos \alpha, \delta(\alpha)=\sin \alpha$, то система эквивалентна обобщённому (сферическому) пятимерному маятнику, помещённому в неконсервативное поле, и обладает полным набором трансцендентных первых интегралов, выражающихся через конечную комбинацию элементарных функций.

Если функция $\delta(\alpha)$ не является периодической, то почти всегда рассматриваемая система является системой с переменной диссипацией с ненулевым средним (т.е. она является “собственно” диссипативной). Тем не менее и в этом случае (благодаря теоремам 2 и 3) можно получить явный вид трансцендентных первых интегралов, выражающихся через конечную комбинацию элементарных функций. Последнее также является новым нетривиальным случаем интегрируемости многомерных диссипативных систем в явном виде.

\section{СПИСОК ЛИТЕРАТУРЫ}

1. Шамолин М.В. Об интегрируемости в трансцендентных функциях // Успехи матем. наук. 1998. T. 53. B. 3. C. $209-210$.
2. Шамолин М.В. Новые случаи интегрируемых систем с диссипацией на касательном расслоении четырёхмерного многообразия // ДАН. 2018. Т. 479. № 3. C. 270-276.

3. Шамолин М.B. Новые случаи интегрируемых систем с диссипацией на касательном расслоении многомерного многообразия // ДАН. 2018. Т. 482. № 5. C. 527-533.

4. Козлов В.В. Рациональные интегралы квазиоднородных динамических систем // Прикл. матем. и механ. 2015. Т. 79. № 3. С. 307-316.

5. Козлов В.В. Интегрируемость и неинтегрируемость в гамильтоновой механике // Успехи матем. наук. 1983. T. 38. B. 1. C. 3-67.

6. Пуанкаре А. О кривых, определяемых дифференциальными уравнениями. М., Л.: ОГИЗ, 1947.

7. Трофимов В.В., Шамолин М.В. Геометрические и динамические инварианты интегрируемых гамильтоновых и диссипативных систем // Фундам. и прикл. матем. 2010. Т. 16. В. 4. С. 3-229.

8. Шамолин М.В. Новые случаи интегрируемых систем с диссипацией на касательном расслоении к многомерной сфере // ДАН. 2017. Т. 474. № 2. C. $177-181$.

9. Шабат Б.В. Введение в комплексный анализ. М.: Наука, 1987.

\title{
NEW CASES OF INTEGRABLE NINTH-ORDER SYSTEMS WITH DISSIPATION
}

\author{
M. V. Shamolin \\ Lomonosov Moscow State University, Moscow, Russian Federation
}

Presented by Academician of the RAS V.V. Kozlov October 3, 2019

Received October 7, 2019

The paper shows the integrability of certain classes of ninth-order dynamic systems that are homogeneous in part, in which the system on the tangent bundle to four-dimensional manifolds is distinguished. In this case, the force fields have various dissipation and generalize the previously considered ones.

Keywords: dynamical system, integrability, dissipation, transcendental first integral. 